\title{
Wie die IT-Revolution die Lohnschere spreizte
}

\section{Marko Mlikota}

\section{Relevanz}

Von 2002 bis 2012 ist in den USA der Medianlohn der untersten $10 \%$ der Lohnskala um 9,5\% gestiegen. Dies im Vergleich zu einem Anstieg von $22,5 \%$ für die obersten $10 \%$. Auf was lässt sich diese sich öffnende Lohnschere zurückführen? Krusell u. a. (2000) zeigen, dass in den 90er Jahren insbesondere die IT-Revolution und die daraus resultierende Nachfrage nach hochqualifizierten Arbeitskräften den Anstieg der Lohnungleichheit verursachten. Was die IT-Revolution in den 90er Jahren bewirkte, stellt sich heute als Herausforderung der Digitalisierung und Automatisierung neu. Eine fundierte Ausbildung ist wichtiger denn je, um in einem sich wandelnden Arbeitsmarkt nicht als Verlierer dazustehen.

Beatrix Eugster

\section{Quelle}

Der nachfolgende Text ist eine Zusammenfassung von: Krusell, Per, Lee E. Ohanian, José-Víctor Ríos-Rull und Giovanni L. Violante (2000), Capital-Skill Complementarity and Inequality: A Macroeconomic Analysis, Econometrica 68, 1029-1053.

Die Einkommens- und Vermögensungleichheit und die damit einhergehenden Verteilungsfragen sind brisante Themen, die sich nicht nur auf die rein ökonomische Debatte beschränken. Die Einkommensungleichheit scheint in den 1970er

M. Mlikota $(\bowtie)$

Universität St. Gallen, St. Gallen, Schweiz

E-Mail: marko.mlikota@student.unisg.ch

(C) Der/die Autor(en) 2018

C. Keuschnigg (Hrsg.), Inklusives Wachstum und wirtschaftliche Sicherheit, https://doi.org/10.1007/978-3-658-21344-2_20 
Jahren aufgrund der IT-Revolution und den angestoßenen Innovationen stark zugenommen zu haben. In diesem Zusammenhang steht das Steigen der Bildungsprämie (skill premium), d. h. des Lohnes von qualifizierteren Arbeitskräften relativ zu dem von weniger qualifizierten und sogar ungelernten Arbeitern, exemplarisch für die sich verändernde Struktur und Dynamik der Ungleichheit.

Studien der 1990er Jahre konnten nicht erklären, wieso die Bildungsprämie stark angestiegen ist, obwohl gleichzeitig auch das Angebot von qualifizierten Arbeitskräften auf dem Arbeitsmarkt relativ zum Angebot von unqualifizierten Arbeitskräften grösser wurde. Sie folgerten, dass eine nicht direkt beobachtbare Änderung in der Art der Produktion von Gütern und Dienstleistungen die Ursache für den Anstieg der Bildungsprämie sein müsse. Diese Änderung in der Produktionsart wurde als bildungs- oder wissensintensiv (skill-biased) beschrieben, weil sie den Einsatz von hochqualifizierten Arbeitskräften verlangt und weniger gut ausgebildete Arbeitskräfte teilweise ersetzt. Krusell und seine Co-Autoren gehen der Annahme des bildungsintensiven technologischen Wandels auf den Grund und analysieren, in wieweit das scheinbar paradoxe Ansteigen der Bildungsprämie durch beobachtbare Variablen erklärbar ist.

Unter einem bildungsintensiven technologischen Wandel werden hochqualifizierte Arbeitskräfte für Firmen im Produktionsprozess immer wichtiger im Vergleich zu ihren weniger gut ausgebildeten Kollegen.

Die Studie beschreibt das Verhalten von Haushalten und Firmen im Produktionsprozess. Dabei weist die Produktionstechnologie eine Komplementarität von Kapital und Bildung auf. Das bedeutet, dass die Firmen bei steigendem Einsatz von wissensintensivem Kapital (z. B. komplexe Maschinen, Lizenzen, oder Patente) auch relativ mehr hochqualifizierte Arbeitskräfte einsetzen. So braucht es z. B. mehr Ingenieure, um komplexe Maschinen zu bedienen, jedoch weniger unqualifizierte Arbeitskräfte, deren Arbeitsleistung durch Maschinen ersetzbar ist. Die erhöhte Nachfrage nach qualifizierter Arbeit steigert den Lohn von hochqualifizierten Arbeitskräften im Vergleich zu demjenigen von weniger qualifizierten Angestellten. Die Bildungsprämie steigt.

Die Forscher nutzen Daten zum Einsatz von Kapital und Arbeit in den USA zwischen 1963 und 1992. Die Schätzergebnisse bestätigen, dass Kapital und Bildung komplementär sind. Die Forscher können mit ihrem Ansatz die Entwicklung der Bildungsprämie im genannten Zeitfenster erklären. Zur Überprüfung ihrer Resultate berechnen sie die Vorhersagen ihres Modells mit und ohne Komplementarität von Kapital und Bildung. Wenn die Komplementarität ausgeschaltet ist, kann nur noch das Angebot von qualifizierter Arbeit die Bildungsprämie beeinflussen. Die Autoren schätzen, dass die Erhöhung des Angebots an qualifizierten 
Arbeitskräften zu einem Rückgang der Bildungsprämie von ca. $40 \%$ geführt hat. Tatsächlich ist die Prämie aber um $18 \%$ gestiegen. Daher muss der technologische Wandel alleine mit der Komplementarität von Kapital und Bildung einen Anstieg der Bildungsprämie von brutto ca. $60 \%$ verursacht haben. Dieser wurde mit der Zunahme hochqualifizierter Arbeit teilweise wieder kompensiert.

Die Bedeutung der Komplementarität von Kapital und Bildung zeigt sich auch in folgendem Szenario. Wäre der Kapitalstock nach 1975 nur so langsam gewachsen wie in der Zeit davor und wäre daher die Nachfrage nach hoch qualifizierter Arbeit nicht so stark gestiegen, dann hätte die Bildungsprämie lediglich um $8 \%$ anstatt um $18 \%$ zugenommen (siehe gestrichelte Linie in Abb. 1). Der Anstieg des Kapitaleinsatzes seit den 1970er Jahren in Verbindung mit einer Technologie, die mehr und mehr auf hochqualifizierte Arbeitskräfte setzt und weniger gut ausgebildete Arbeiter immer mehr durch Maschinen ersetzt, dürfte den Anstieg der Bildungsprämie verursacht haben. Diese Erklärung greift für die Zeit bis zu den 1990er Jahren, ist aber vermutlich auch heute noch gültig.

Der erhöhte Kapitaleinsatz in Verbindung mit einem bildungsintensiven technologischen Wandel hat den relativen Lohn von hochqualifizierten Arbeitskräften in den 30 Jahren von 1963 bis 1992 um 60 \% erhöht.

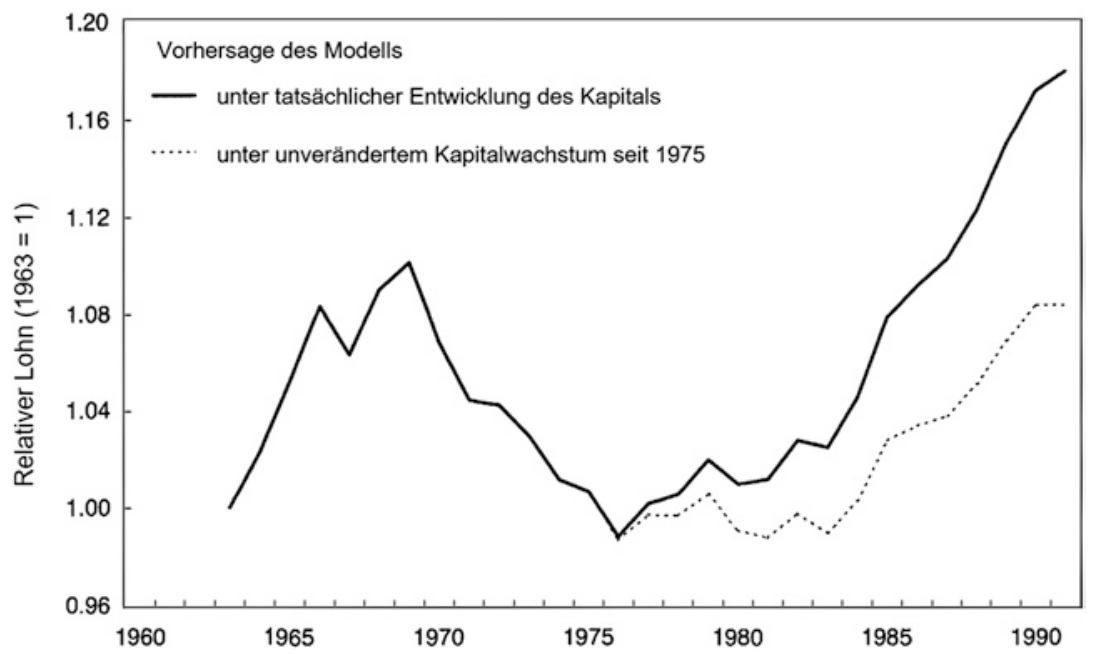

Abb. 1 Beitrag des Kapitalwachstums zum Steigen der skill premium (Quelle: Krusell u. a., 2000, 1045) 
Eine konkurrierende Erklärung für den Anstieg der Bildungsprämie könnte sein, dass hochqualifizierte relativ zu weniger qualifizierten Arbeitskräften produktiver und deshalb auch besser entlohnt werden. Leider sind die Produktivitätsniveaus für qualifizierte und nicht qualifizierte Arbeitskräfte nicht direkt beobachtbar, was das Überprüfen dieser Hypothese erschwert. Die Forscher zeigen jedoch, dass der Produktivitätsunterschied um den Faktor 30 gestiegen sein müsste, was als wenig realistisch erscheint.

Das Forschungsergebnis könnte für Entscheidungsträger in Wirtschaft und Politik hinsichtlich der Ungleichheitsdebatte äusserst relevant sein. Es stellt die Effektivität von Handelsbarrieren zum Schutz von einheimischen, weniger qualifizierten Arbeitnehmern in Frage. Diese konkurrieren nicht nur mit ausländischen Niedriglohnarbeitern, sondern auch mit immer effizienteren und billigeren Kapitalausstattungen, die mehr Qualifikation verlangen. Eine bessere Ausbildung scheint die einzige nachhaltige Möglichkeit zu sein, um zu verhindern, dass diese Arbeitskräfte mehr und mehr von Robotern und Computern verdrängt werden. Die Studie zeigt damit die erhöhte Bedeutung von Bildung und Qualifikation in einer innovativen Wirtschaft auf. Eine Bildungsoffensive mit einem erhöhten Angebot von hochqualifizierten Arbeitskräften kann einer zunehmenden Ungleichheit effektiv entgegenwirken.

Open Access Dieses Kapitel wird unter der Creative Commons Namensnennung 4.0 International Lizenz (http://creativecommons.org/licenses/by/4.0/deed.de) veröffentlicht, welche die Nutzung, Vervielfältigung, Bearbeitung, Verbreitung und Wiedergabe in jeglichem Medium und Format erlaubt, sofern Sie den/die ursprünglichen Autor(en) und die Quelle ordnungsgemäß nennen, einen Link zur Creative Commons Lizenz beifügen und angeben, ob Änderungen vorgenommen wurden.

Die in diesem Kapitel enthaltenen Bilder und sonstiges Drittmaterial unterliegen ebenfalls der genannten Creative Commons Lizenz, sofern sich aus der Abbildungslegende nichts anderes ergibt. Sofern das betreffende Material nicht unter der genannten Creative Commons Lizenz steht und die betreffende Handlung nicht nach gesetzlichen Vorschriften erlaubt ist, ist für die oben aufgeführten Weiterverwendungen des Materials die Einwilligung des jeweiligen Rechteinhabers einzuholen.

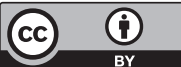

